\title{
WALKING THROUGH THE CANTOR'S PARADISE AND ESCHER'S GARDEN: EPISTEMOLOGICAL REFLECTIONS ON THE MATHEMATICAL INFINITE (I)
}

\author{
J.L. Usó-Doménech and J. Nescolarde-Selva \\ Department of Applied Mathematics. University of Alicante. Alicante. Spain.
}

\section{H. Gash}

Education Department. St Patrick’s College, Dublin City University. Dublin. Ireland.

I heap up monstrous numbers,

Pile millions upon millions,

I put aeon upon aeon and world upon world,

And when from that awful height

Reeling, again I seek thee,

All the might of number increased a thousand fold

Is still not a fragment of thee.

I remove them and thou liest wholly before me. (Albrecht von Haller)

Abstract

Infinity is not an easy concept. A number of difficulties that people cope with when dealing with problems related to infinity include its abstract nature, understanding of infinity as a never ending process, understanding infinity as a set of an infinite number of elements and understanding some wellknown paradoxes. Infinity can be understood in a number of ways some of which are incompatible, and involve value judgments or assumptions that are neither explicit nor desired. In its definition, we distinguish several aspects, teleological, artistic (Escher, 2000), some essential, some potential, and others actual. Cantor’s work on set theory is linked to infinity and has implications for belief in God. 
Keywords: axiomatic formal system, infinity, metaphysics, paradoxes, set theory, transfinite regions

\section{INTRODUCTION: INFINITY AND TRANSCENDENCE}

Infinity does not exist in the material world. Although the number of elementary particles, atoms, stars, galaxies, etc. is immensely great, it is not infinite. However, infinity is a concept that is handled not only in mathematics but in theoretical physics. Is it "real", or is it a belief that only exists in the human mind? For Aristotle there was only infinite potential, i.e., the limit of a sequence and he denied actual infinity, which for Spinoza is infinite reason. Cantor "discovered" infinity in his work on set theory and transfinite regions. Although mathematicians seek to render unnecessary the metaphysical implications of transfinite theory, these implications are linked to that central fundamental human belief: the existence of God. An aim of this paper is to show this in both Cantor's and associated work.

Infinity is that which is without (cannot have) term or end (Latin infinitus). Now, consider some problems. Is it possible to shorten the infinite, and what is the result? Can we have half of infinity? How many integers are there? How many even numbers are there? In each of these cases we have an infinite number, that is, the same in each case, as by definition nothing can be greater than infinity, no set can it be greater than the set of even numbers.

But it seems obvious that the set of integers exceeds that of even numbers. If they are different, we would have to wonder how big the difference is. In this case, is the set of odd numbers infinitely large? The search to understand infinity has been constant in mathematical and philosophical thought, from its origins in Archaic Greece to contemporary science. We need to explain the world around us. We are perturbed by the 
possibility of a point in space beyond which there is absolutely nothing, not even emptiness. What sort of world system do we believe in?

The question “what is infinity?” remains intriguing for mathematicians We live in a world that usually offers tangible things, things we can measure that have a beginning and an end, finite things. However, for some things the boundaries are blurred, and hard to pin down. Time is one example, our life is finite, we are born and die, but when a creature dies, the weather continues impassively, and seems to have no end ... which is eternal. In every culture there seems to be a concept of endless time. An immortal infinite existence is a quality frequently assigned to divine beings. The space around us seems without any defined boundary, we look to the horizon but if we move it remains equally distant. The same is true when looking at the stars, though not visible to the naked eye, we know that beyond the galaxies are further clusters of galaxies and, although the real world can seem finite, our imagination is not afraid to keep looking. Something in our brain tells us that space goes on forever, and if we say it does not, the question always arises, what lies beyond? Importantly, infinity raises issues about how concepts work and what we can kngw in our experience. Ideas about the unattainable, immortality, infinite time and space are amongst the crucial issues raised in understanding our humanity.

When we talk of the infinite, we tend to think of huge things but infinity can also be reached via smaller and smaller elements. Consider the Russian dolls (matriuskas) that are hollow inside, so that there is a new doll inside, and then in turn another, and another ... In this case the process would end by the human inability to create microscopic objects, but with numbers this limitation vanishes. Given a number, one is always able to get a smaller one. 
One progressive approach to mathematical thinking is structuralist. Mathematics has been defined as the study of species of structures. The idea of mathematics as a "free creation" allows the thinker to propose alternative orders to that which previous mathematicians had considered fixed immutable laws of God. The aim is to capture, in one stroke, the entire nature of things, an explanation of the concept of structural species is one in which our universal system is a particular example. Mathematicians persist in thinking of a point as a dimensionless place in an n-dimensional manifold. Any manifold, upon this thought will have an infinite number of points. An "axiom of transcendence of the zero point ${ }^{1 "}$ in relation to the universe of any three-manifold implies that one can not specify a dimensionless point in a multi-dimensional space (Moore, 1991). The notion of a point as a non-dimensional point in an n-dimensional manifold is contradictory and confusing (and that is why sets behave paradoxically when trying to manipulate mathematically under the assumption of zero locations in the universe of a set). The confusion is resolved by specifying a dimensional point in a multi-dimensional space. This alternative position can be formalized in what is called the axiom of transcendence: a point of non-transcendent dimension in a universe of any type. The meaning of the axiom of transcendence is due to the position that a nondimensional point specification is essential for a rigorous definition of a continuous process, so that a space-time continuum is an unrealizable ideal notion for any actor. The idea of the infinite takes a central place in thinking about transcendence. In it the identity model is broken, emphasizing the capacity of a thought to contain more than

\footnotetext{
${ }^{1}$ Any manifold, in this thinking, will have an infinite number of points. An "axiom of zero-point transcendence" with regard to the universe of any dimensional manifold implies that you cannot specify a non-dimensional point on a dimensional manifold. The notion of a point as a non-dimensional location on an -n- dimensional manifold is contradictory and leads to confused mathematical thinking. You can only specify a dimensional point on a dimensional manifold. This alternative position may be formalized in what is termed the Axiom of Transcendence: a non-dimensional point is transcendent of the universe of any manifold.
} 
that which is thought. But a new break is needed: out of being (Levinas, 1977) as a place of transcendence, and a time that is not my time. The model is the Cartesian model of the relationship that I established with the Infinite, in which both terms are maintained with no possibility of being reduced. But this relationship is complex and is neither an occasion for delirium or insanity as loss of self, not is it a demonstration of the existence of God.

\section{PARADOXES AND PARADOXES OF INFINITY}

The concept of a paradox can be understood as one of the following:

1) A contradictory statement appears to be true.

2) A statement exhibiting inexplicable or contradictory aspects or qualities.

3) A contradictory statement based on valid reasoning andlogical assumptions.

A fallacy paradoxes contain arises out of the circumstance that there are defined or presupposed wholes, whose existence would imply the existence of new elements definable only in terms of the whole (Gödel 1981: 51). Consider: All propositions are either true or false. We want to highlight the following: to prevent the emergence of paradoxes, Russell (Whitehead and Russell, 1910-1927) proposed his vicious circle principle that avoiding vicious circles involves simply showing that nothing is done that involves all of a collection being considered as a member of the collection, because if a supposed collection has a total and one tries to stipulate new members only definable in terms of that whole, then the collection in question is no longer a whole. It will have to include the new member if it wants to be a whole. It is as if we could not reach the whole, since we are always pushing one step further: it is always one step ahead. These wholes that 'escape us' are what Russell called illegitimate totalities. This does not mean, however, we cannot talk of wholes, or legitimate wholes. Legitimate wholes are 
those that have been limited to complete their entirety, and any subsequent totality that relates to that whole must fall entirely outside the first whole. We can ask this question about the vicious circle principle: why should we accept it? In contrast to what happens in the case of the axiom of reducibility, Russell offers us a number of reasons for acceptance. Its usefulness in terms of destruction of the paradoxes seems to be enough to justify its existence. This is something that seems perfectly obvious. Indeed, the continent must be larger than the content. The paradox we want to analyze is that of Russell. This paradox can be explained very simply as follows: There are some classes that contain themselves and others that do not contain themselves. Examples of the latter are the class of all math professors and we have no major problem with it. An example of the first is the class of all the ideas presented in this paper. This class contains among its objects the same class, because the class of all the ideas presented in this paper is an idea that is in this paper. The question is this: is the class of all classes that do not contain themselves a member of itself? If it is not a member of itself, should be, because the class includes all classes that are not members of themselves, and if instead it contains itself it should not contain itself, since the class is only of classes that do not contain themselves. This is Russell's paradox. Now let's see what happens in the case of the solution to it. Russell tells us that a class is an object derived from a function $(\varphi)$ and assumes the role for which it is derived. $\varphi \mathrm{x}$ assumes the function $\varphi^{*} \mathrm{x}$. Therefore, a class cannot significantly be the argument of the function to which it relates. We can then say that a class is not admissible as an argument of the function to which it is related. Thus, there is no question of the class of all classes that are members of it. If there is no question of this class, it is impossible that the paradox arises because the paradox we are talking about depends on how one can talk about the class mentioned. 
For Russell, all paradoxes arise from the ambiguity of words like falsehood, class, name etc ...The paradox brings out this ambiguity in these words and highlights the appearance of self-contradiction present in all paradoxes. However, Russell also thinks that this ambiguity, or rather, these ambiguous words should not be sacrificed. They are necessary in mathematics and mathematical logic, since these fields often need to be used with a multitude of ideas to be able to interpret an infinity of different determinations. Ambiguity in these fields allows the same reasoning to be applied to a large number of cases. What was clear to Russell is that this ambiguity should be kept heavily guarded and watched over so that it cannot exercise its influence without critical examination.

\subsection{Some paradoxes on infinity}

Historically, the infinite has always been a source of difficulties and paradoxes. From the point of view of mathematical theory, many of the difficulties of infinity have been resolved as that concept has been defined as a mathematical object with its own field of operation; particularly in the work of Bolzano, discussed in his book appropriately entitled Paradoxes of the Infinite (1950).

Example 1: Gabriel's trumpet, or Torricelli's trumpet is a funnel-shaped surface (or trumpet). It starts broad and narrows quickly, but never closes - i.e., to infinity. The surface of the trumpet is infinite, but the volume that it contains is not infinite ${ }^{2}$. Suppose we paint the trumpet gold inside. The surface is infinite, and so an infinite amount of

\footnotetext{
${ }^{2}$ If the surface has an infinite surface it should have an infinite volume. But when the calculation is done, it turns out that the volume of this object is $\Pi$ ! It is therefore an object with an infinite surface but with a finite volume.
} 
paint is required for the outside. However, a finite amount of paint, which corresponds to the volume of the trumpet is required to paint the inside. Then which is more confusing: the idea that an infinite surface implies a finite volume or the idea that a finite amount of paint covers an infinite surface? Galileo's disciple Torricelli was the first to think of this problem, which he found so extraordinary that at first he thought he had done something wrong. Interestingly, in the case of the famous paradoxes of Zeno, there also arises a conflict between two components of a different nature. Recall the essence of these paradoxes (which can be considered as paradoxes of the infinitely small): Zeno of Elea (c. 450 BCE) questioned the belief that, "the sum of an infinite number of quantities can be made as large as desired, even if each quantity is extremely small $(\infty x \quad \varepsilon=\infty)$, and also that the sum of a finite or infinite amount of zerodimensional numbers is zero." His arguments emphasize the difficulty arising from understanding a line as consisting of points (the problem of the relationship between the continuous and discrete). Zeno posed paradoxical situations in which this is illustrated, first, that if continuous variables (such as time and space) were infinitely divisible, then movement could not exist; but on the other hand, if space was infinitely divisible (by the existence of indivisible parts), then there could be no movement! Aristotle believed that one of the first problems is that when Zeno's paradox suggests that the infinite division of space requires an infinite time to complete, he mixes two types of infinity: infinite divisibility of space, and an infinite length of time. For Aristotle, Zeno's paradoxes reveal the incoherence of something which is divided into an infinite number of parts (which involves the construction of an actual infinite). So, Aristotle argues that it is false to assume that the continuous consists of indivisible elements. Zeno's paradoxes arise when mixing the discrete with the continuous (by applying a number to an extent), and Aristotle's solution was to separate the discrete from the continuous. 
We live in a fractured world or quantized space-time where and due to zero point convergence (energy), it is difficult to specify a manifold (space). Zeno in his paradoxes had implicitly answered the question whether space-time is continuous or discontinuous. The physical sciences today seem to have responded similarly to this question with the notion of a "Planck unit" of space-time as a unit with a fundamentally different zero point of space-time. Calculation does not resolve the paradoxes of Zeno because an extremely small transformation in space-time is not yet a zero point, but a fragment of a whole which has, in turn, an infinite number of points. Infinitesimal analysis, the most favorable evaluation, only approaches the notion of continuous movement and is only relevant when the theories are expressed in terms of classical concepts of physics. However; quantum theory says that movement is essentially discontinuous. The dichotomy paradox, which, in our opinion, summarizes the other paradoxes of Zeno, as stated by Aristotle, postulates a continuum space-time of an infinite number of points, and then goes on to question how movement might be possible in such circumstances, as anybody moving in continuous space-time must pass through an infinite number of intermediate points in any interval of space. The solution that we can reasonably propose to Zeno's paradox is similar to the Achilles solution in Lewis Carroll (1985) ${ }^{3}$ : for Achilles to catch the tortoise, he must, at a point in arbitrary time, equate a different point of zero fraction of space with a convergence zero point of space, then take advantage of this logical trick to reach the tortoise.

Example 2: Galileo's paradox: although not all numbers are square numbers there are not more numbers than square numbers. This paradox is a demonstration of one of the

\footnotetext{
${ }^{3}$ http://www.ditext.com/carroll/tortoise.html
} 
surprising properties of infinite sets. The paradoxical character arises by calling into question the principle that the whole is greater than its parts.

In his final scientific work, Two New Sciences, Galileo Galilei made two apparently contradictory statements about positive integers. First, some numbers have the property of being a perfect square, while others do not. Therefore, the set of all numbers, including both square and non-square, has to be larger than the set of squares. However, for each square there is exactly one number which is the square root, and for each number there is exactly one square. Therefore, there cannot be more of one type than another. Galileo concluded that the concepts of less than, equal and greater applied only to finite sets, and could not be applied to infinite sets. In the nineteenth century, Cantor, using the same methods, showed that although Galileo's result was correct when applied to integers, or even rational numbers, the general conclusion was not true: some infinite sets are larger than others, in the sense that they cannot be related in one-to-one correspondence.

\section{THE CANTOR'S PARADISE}

"No one will drive us from the paradise which Cantor created for us."(Hilbert)

Ontological arguments supporting Cantor's theory of transfinite numbers are unknown to most of the mathematical community. Current mathematical discussion has limited the concept of infinity to potential and actual infinity. The philosophical-mathematical infinity spectrum contains in Cantor's work a metaphysical variant, which is called "Absolute Infinity". The transfinite theory of sets is located between the absolute infinite and the finite. However, the fact that transfinite regions are accessible to human 
knowledge (mathematical) is an argument against the Kantian thesis ${ }^{4}$ that man cannot rise above his own finite position with respect to knowledge, that is, it is not possible to leave the limits of possible experience by finding a knowledge of the infinite. Human beings, according to Cantor, are unable to have a proper knowledge of absolute infinity (God). However, this does not mean that man has no proper access to the infinite. Indeed, the formation of transfinite mathematical concepts must prove that the Kantian limitation is unnecessary. To reinforce this argument, Cantor resorts to arguments of rational theology, one that Kant had totally eliminated from the field of possible knowledge. This is based on Cantor's impatience and lack of understanding in relation to the mathematical "horror infiniti" that in fact, was just a critical reserve against a certain type of philosophy introduced in mathematics. Actually, Cantor uses rational theology and philosophical reflections to pass from the conceptualization of the transfinite, to its theoretical relevance to reality: Cantor distinguishes in mathematical concepts, especially in regard to the concept of number, two realities: intrasubjective or immanent reality and extrasubjective or transcendent reality ${ }^{5}$. The immanent reality of certain concepts is understood to be well defined. In addition to this, that the concepts also have transcendent reality means they have to be considered as an expression or

\footnotetext{
${ }^{4}$ Kant (2011) believed that reason is always looking for the condition or foundation of things. Scientific research rests on thîs desire of Reason to understand the causes, conditions or foundations of phenomena. But if the spontaneous functioning of Reason is not limited by critics, how will people think about the conditions or foundations of phenomena: in relation to our psychic life, the physical world and the foundation of all phenomena, both physical and mental. When Reason acts in an uncontrolled way, it will end up thinking of the traditional objects of metaphysics: the soul, the world as a whole and God. Kant believed that the use of reason, which he calls dialectical - is inadequate and leads to fallacies and contradictions.
}

\footnotetext{
${ }^{5}$ Some Jewish writing (Kaplan 1990; Saks 1990, 2002; Schochet 1979) refer to different levels of infinite spiritual worlds, an idea similar to Georg Cantor's idea that there is an infinite sequence of (mathematical) infinities: $\aleph_{0}<\aleph_{1}<\aleph_{2}<\ldots$. Cantor's demonstration that the infinite cube contains exactly the same number of points as in the one-inch cube helps to understand "the paradox of God's simultaneous transcendence and immanence"
} 
image of processes and relationships concerning our experience of the external world, by the intellect. Epistemological conceptions by which Cantor distinguishes between a transcendent reality and an immanent reality are difficult and mysterious. However, in Cantor the requirement "mathematically free", turns transfinite theory into a possible mathematical object, even if only to ensure that the concepts are well-defined modifications of the substance of the human spirit.

The point of view of Cantor offered in many mathematical disciplines (theory of functions, topology, etc.) as new fields of activity, presented new problems that on finite bases had not been resolved or would have needed a very subtle and careful treatment. What is important is not that certain demonstrations were correct or not, but whether they should be used or not. Cantorian criticism of set theory is very similar to Kant's critique of metaphysics in the Transcendental Dialectic. In the same way that what matters to Kant is not to attack demonstrations of rational theology, cosmology and psychology as correct proofs, but rather the question of the meaning of the demonstration in classic areas of metaphysics. In the same way in the discussion on sets and transfinite powers, Cantor was not so interested in the various mathematical demonstrations that are not correct, but rather in opening a possible field of scientific problems. Whoever accepts Cantor's speculation regarding the concept of infinity as the foundation of a new field of mathematical activity, cannot, as a mathematician, refuse to accept the propositions and demonstrations of transfinite theory. As Cantor's set theory led to certain contradictions similar to some antinomies analyzed by Kant, it is no surprise that Cantor had to have recourse to rational theology and cosmology.

The conceptual world of set theory has come to be the universal basis of current mathematics, only under the assumption that, as a mathematician, it must be released from the corresponding metaphysics, i.e., you can talk as Cantor but not think as Cantor. 
You may walk in Cantor's paradise without having to worship the God who guarantees this metaphysical paradise as Platonic or empirical, pursuant to its peculiar inclinatio infiniti $^{6}$. However, Hilbert believed he could separate philosophical sophistry from mathematics. His solution was a formal or theoretical structural interpretation of mathematics. This point of view made it possible for relatively arbitrary (as to their content) systems of propositions to be transformed into a legitimate object of mathematical research. This is regardless of the precise meaning of the terms that have content, and conceiving these terms formally, through forms of statements of the system of propositions. It is a premise that the investigated system of propositions is free of contradictions, or, at least, that there has not been, until now, any contradiction. Therefore, there are formal axiomatic systems, those that Hilbert states are starting points for mathematical research, and therefore are definiens of mathematical objects. Mathematics no longer makes categorical statements about what nature is, but only hypothetical statements based on what is, when there is a certain formal structure. Thus, a mathematician can eliminate discussion of the metaphysical foundations of Cantor's set theory, and at the same time, keep this set theory in the form of a formal axiomatic system, as a foundation of mathematics. Thus, the antinomies of set theory give no reason to banish the conceptual world of Cantor from the mathematical world; rather they led to the replacement of Cantor's call for a naive set theory by a formal axiomatic system, which so far has not led to the appearance of any contradiction. Likewise, the axiomatic-formal interpretation of the whole of mathematics is not without problems. First, it gives to mathematics an immense amount of possible objects, whose designation as "formal theories" can only obscure the fact that for many of them, it is

\footnotetext{
${ }^{6}$ For any current mathematical metaphysics of Cantorian infinity is more suspect than the metaphysics of philosophers.
} 
not possible to substantiate a theoretical nature, without resorting to Cantor's metaphysics.

There is another argument that one can understand relatively formal theories as theories of explanation or description. This justification is based precisely on the concepts and "realities" of set theory, which are in Cantor's paradise. The Cantorian ontological regions provide sufficient possibilities of interpretation to almost justify arbitrary formal theories. This statement is more trivial than it seems, since the syntactic possibilities that should be adjusted for the formation of formal theories, harmonize with reasonable accuracy Cantor's conceptions about the ontology of sets. If the axioms of set theory represent the ontological principles of a region of entities and facts, which are legitimate objects of human knowledge, then we could understand axiomatic set theory as a formalized theory in this region. But this still does not exclude another possibility: the theoretical-applied interpretation of conceptual formalizations of set theory. In this case, we should interpret the axioms of set theory as mere assumptions that are justified by scientific applicability. Those who do not believe in Cantor's paradise but appreciate the heuristic value of conceptual formations adopt this position.

The position of theoretical application thus obtained denies any fundamental difference between laws and mathematical models and concepts and axioms (on a foundation of set theory), and rejects another explanation for this difference. Set theory would be a physical hypothesis among others, or would not clarify any difference in conflict with the "facts" of the patent difference between mathematics and physics. The second point of view would be unworthy of a theoretical scientist, even if he had to give up this position, leaving the conceptual world of Cantor. For example, in the case of a "hypothetical" interpretation of the mathematical foundations of set theory, there has to be a temporary emergency solution. This is true more so as there is a concurrent 
foundation of mathematics, which not only avoids the highlighted difficulty, but also restores the theoretical character of mathematics in an Aristotelian sense ${ }^{7}$. This starting point, finite, constructive or operative, defines mathematics as a theory of actions or of more schematic and perceptible operations, and is represented by the reflections of the later work of Hilbert and Wittgenstein, Dingler and Lorenzen.

Mathematical assertions do not refer to empirical facts, but patterns of action, and thus assume "experience" only as an exercise. With this, the mathematician gets information about the "foundation" of arithmetic facts, insofar as that these may be referred to as the simplest of all systems: do it and do it again, based on the above result, as concrete "objects" of arithmetic. If instead one desires to continue the current trend in the interpretation of arithmetic, we should refer to mystical entities as the set containing as its only element the empty set, or the set of all sets of an element ${ }^{8}$. Or submit an axiomatic formal system as the "beginning" of arithmetic, for example, a form of Peano axiomatic system. In the first case, one is forwarded to Cantor's metaphysical speculations regarding the foundation of the ontology of sets; in the second case, one is

\footnotetext{
${ }^{7}$ The concept of theory, insofar as that it has determined the history of philosophy and science, was first used by Aristotle. It is a fundamental component of the meaning of the Aristotelian terms $\vartheta \varepsilon \omega \rho \imath \alpha$ and $\varepsilon \pi \sigma \tau \eta \nu \eta \vartheta \varepsilon \omega \rho \eta \tau \imath \chi \eta$, which point out the difference between knowing about the logic or explanation for a particular state of affairs and the pure verification of this state of affairs. Theories, in the Aristotelian sense, are answers to questions that begin with the interrogative "why" ( $\delta i \alpha \quad \tau \imath$ ). Using an Aristotelian example (Aristotle, 2002), a theorist is one who knows why fire is hot, unlike the purely empirical person, who simply knows it's hot. In Aristotelian philosophy theories are referred to as "theories based on principles" or theories of explanation. Theoretical knowledge is liberated by definition, according to Aristotle, of any need for justification. In this way, the same right could be granted to formal theories, whether or not they legitimately belong to the tradition of theory. Indeed, Aristotle provides a justification in the Nicomachean Ethics (2011). Aristotle distinguishes theoretical science, from logic which is the essential upgrade of reason. For this reason, theory, especially metaphysics, such as research and knowledge of the latest principles, may be the perfection of praxis of man as man and leads to happiness that is linked to this praxis.
}

\footnotetext{
${ }^{8}$ Both are ontological interpretations of set theory of number 1.
} 
again faced with the difficulty of being unable, under the arguments already discussed, to provide an immanent formal justification of formal arithmetic, while theoretical science, and therefore the problem of the foundation of arithmetic is not solved, it is just left out.

One could insist on the heuristic advantages of a foundation based on set theory with respect to a constructivist point of departure, which until now has been poorly explained. If the Platonism of set theory is understood as a theory based on implications in the sense defined above, it could have essentially specific implications i.e., that constructively are neither achievable nor irreplaceable, but nevertheless indispensable in the fields of application of mathematics. There is no reason for conflict as the natural sciences are possible, especially physics, without the Platonism of set theory as a basic hypothesis $^{9}$. By contrast, for the mathematical tools of theoretical physics, there is a constructive basis that provides all the essentials. The simple fact that Physics uses the transfinite, its own interpretation of the ontology of set theory, the infinitesimal calculus, and does not use its constructivist interpretation, is not demonstrating any necessary link.

From here one can only infer that the task of preparing a comprehensive and sufficiently constructivist elemental analysis system, and introduce the mathematician knowledge of the physical, has not yet been realized. If this were done, it would have placed an archangel before the door of Cantor's paradise.

\subsection{Some problems}

\footnotetext{
${ }^{9}$ As regards the physical, such was the opinion of Cantor. He refers to physics, not to support the assumption of a transfinitum in natura naturata, but refers to biological and psychological data.
} 
1) We will introduce some preliminary concepts. We say that a number is definable if there is any property that defines it. $\pi$ and $8 / 5$ are definable numbers. In reality all rational numbers are definable, although some irrational are also definable, like the aforementioned $\pi$. Other well known irrational numbers include the number $e$ (Euler's Number), the square root of two and the golden ratio ${ }^{10}$. We include in this concept, all conceivable, past, present or future definitions. That is, a definable number is any one that has ever been defined or that could ever be defined at some point in the future (whether that definition is really ever written, or simply expressible).

No definable numbers are ineffable. That is, ineffable numbers are those numbers that have never been defined and never, even in theory, can be defined at any time or place.

Georg Cantor proved that the set of real numbers is uncountable. That is, it is impossible to establish a one-to-one mapping between the set of real numbers and the set of natural numbers. The set of real numbers has an order of infinity higher than the set of natural numbers.

Theorem 1: The set of all possible definitions is countable.

Proof

The order of infinity of this set is the same as that of the set of natural numbers. Therefore, in a well defined sense, there are more real numbers than possible definitions

\footnotetext{
${ }^{10}$ In Mathematics, two quantities are in the golden ratio if their ratio is the same as the ratio of their sum to the larger of the two quantities. The figure on the right illustrates the geometric relationship. Expressed algebraically, for quantities $a$ and $b$ with $a>b, \quad \frac{a+b}{a}=\frac{a}{b} \stackrel{\text { def }}{=} \varphi$ where the Greek letter $\varphi$ represents the golden ratio. Its value is: $\varphi=\frac{1+\sqrt{5}}{2}=1.6180339887 \ldots$
} 
and therefore it is impossible that there is a one to one correspondence between real numbers and their definitions (past, present or future).

We have demonstrated that there are ineffable numbers (in fact, that there are an infinite number of ineffable numbers). There are no examples. By its very nature, it is impossible to specify a particular ineffable number. Every number we are able to mention is inevitably definable.

Theorem 2: The sum of an ineffable number and a definable number is an ineffable number.

\section{Proof}

Let $\mathrm{x}$ be an ineffable number anyone and $\mathrm{q}$ be a definable number. We have to prove that $\mathrm{z}=\mathrm{x}+\mathrm{q}$ is ineffable.

Suppose, by the absurd, that $\mathrm{z}$ was definable. Then, since $\mathrm{x}=\mathrm{z}-\mathrm{q}$, then $\mathrm{x}$ would definable because it could be defined as: "It is the result of subtracting the number that satisfies (copy here the definition of z) minus the number that satisfies (copy here the definition of $q$ ).

This contradicts the assumption that $\mathrm{x}$ is ineffable. Thus $\mathrm{z}$ is also ineffable.

Now, what do we mean when we say in a demonstration " Let $\mathrm{x}$ be any ineffable number?”

What do you mean "any"? It is usually understood that the use of the word "any" indicates that what is being done is "generic reasoning," that is reasoning that can be 
repeated in every particular case. As it were: "you replace $x$ by any ineffable number and you will see that everything that is said is true afterwards."

But ... how can such an interpretation be accepted in this case if it is impossible (and will always impossible) to take even a particular example? Is this a valid proof of Theorem 2? Does it make sense of the concept of ineffable numbers, although set theory allows us to prove that there exists an infinite number of such numbers?

2) In the theory of Cantor, two infinite sets have the same cardinal number if for any element of one set there corresponds an element and only one of the other set. Any set whose members can be matched with the set of positive integers is given the cardinal number $\aleph_{0}$. Thus, the set of perfect squares has for cardinal $\aleph_{0}$. Correspondence can be represented by:

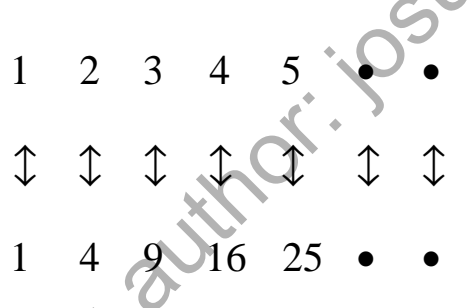

Each number $\mathrm{N}$ corresponds to a number $\mathrm{N}^{2}$ and vice versa. Although the set of squares represents a subset of the set of integers, it has the same cardinality. In the theory of infinity, often the whole is not greater than one of its parts. It is known that the set of all points on a straight line, the rational and irrational numbers, each have a cardinal number that is greater than $\aleph_{0}$. Using his diagonal procedure, Cantor showed that in this set of real points there is no correspondence between each member and an integer. The set has cardinality $2{ }^{\aleph_{0}}$.

If a determinant of n-order is developed in an algebraic polynomial, it contains $n$ ! terms. A determinant is defined as the limit of a determinant of n-order when $n$ tends to 
infinity and is a perfectly respectable mathematical entity; it is known that its development contains $2{ }^{\aleph_{0}}$ terms. What is not known is how to build a biunivocal correspondence, i.e., term to term, between this development and the $2^{\aleph_{0}}$ points on the straight line (Goodman, 1948).

3) This is one of the most famous unsolved problems is Cantor's continuum hypothesis (Manin, 1981): $2^{\aleph_{0}}=\aleph_{1}$. : the smallest transfinite number greater than $\aleph_{0}$ is the number of points on a straight line. There is also a generalization of the continuum hypothesis called the generalized continuum hypothesis (GCH) which says that for all ordinals $\alpha$, $2^{\aleph_{0}}=\aleph_{\alpha+1}$. The unresolved problem is to prove that this hypothesis is true or that it is not. Cantor thought was true. The proposition is equivalent to the following: all infinite subsets of a continuum have either the power of the set of integers, or the power of the entire continuum. However, Gödel (1947) adds some considerations: a superior limit cannot be assigned to the power of the continuum. It is not known whether this power is regular or singular, accessible or inaccessible. Gödel was inclined to think that the hypothesis will ultimately be false. As the reason he gives the extremely unexpected and implausible nature of the consequences it entails. But the fact that a theorem is special andincredible is not sufficient to demonstrate its falsity. Mathematics is full of perfectly yalid things that intuitively might find impossible results. However, as knowledge progresses doubts arise.

An example of the strange and wonderful properties of this hypothesis is that the relationship $2^{\aleph_{0}}=\aleph_{1}$. results from the following proposition: three sets $E_{i}(i=1,2,3)$ can be defined whose Euclidean plane is their union, and three straight lines $D_{i}(i=1,2,3)$ in the plane such that the set $E_{i}$ cut all lines parallel to $D_{i}$ in a finite number of points. It 
can be shown that a proposition of the same kind, but considerably less exacting, also contains the continuum hypothesis.

Questions are raised for those who wish to walk in Cantor's paradise.

\section{REFERENCES}

Aristotle. 2002. Aristotle's Metaphysics. Trans. Joe Sachs. 2nd ed. Santa Fe, N.M.: Green Lion.

Aristotle. 2011. Nicomachean Ethics. Translated by Bartlett, Robert C.; Collins, Susan D. Chicago: University of Chicago Press.

Bolzano, B. 1950. Paradoxes of the Infinite. New Haven: Yale University Press.

Carroll, L. 1895. What the Tortoise Said to Achilles. Mind 4, No. 14: 278-280.

Escher, M.C. 2000. Estampas y dibujos. Ed. Taschen, Colonia. (In Spanish).

Gödel, K. 1947. What is Cantor's continuum problem? Amer. Math. Monthly.Vol 54, p 515.

Gödel, K. 1981. La lógica matemática de Russell. Cuadernos de Filosofía y Letras. Vol.4 no. 1-2,.Universidad de los Andes, Bogotá. (In Spanish).

Goodman, A.W. 1948. The number of terms in the expansion of an infinite determinant. Amer. Math. Monthly. Vol 55, p 419. 
Kant, I. 2011. The Critique of the Pure Reason. Translated by J.M.D. Meiklejohn. Pacific Publishing Studio.

Kaplan, A. 1990. Innerspace. Brooklyn, NY: Moznaim

Lévinas E. 1977. Totalidad e infinito. Ed. Sígueme. Salamanca. (In Spanish).

Moore, A.W. 1991. The Infinite. Routledge; London and New York

Saks, T. V. 1990. Applications of mathematical infinity in Jewish philosophy. In H.

Branover, A. Gotfryd, S. Lipskar (Eds.), Fusion: Absolute standards in a world of relativity, pp. 123-142. Jerusalem, Israel: Feldheim.

Saks, T. V. 2002. Different levels of infinity in Torah and mathematics. B'Or Ha'Torah, 13E, 113-120.

Schochet, J. 1. 1979. Mystical concepts in Chassidism. Brooklyn, NY: Kehot Publication Society.

Whitehead, A.N. and Russell, B. 1910-1927. Principia Mathematica. Cambridge University Press. Cambridge 